\title{
Vitamin-K-Dependent Protection of the Renal Microvasculature: Histopathological Studies in Normal and Diseased Kidneys
}

\author{
Fang-Fei Wei ${ }^{a}$ Nadja E.A. Drummen ${ }^{b}$ Lutgarde Thijs $^{a}$ Lotte Jacobs ${ }^{a}$ \\ Marjolein Herfs $^{b}$ Cynthia van't Hoofd ${ }^{b}$ Cees Vermeer ${ }^{b}$ \\ Jan A. Staessen ${ }^{a, b}$ \\ aStudies Coordinating Centre, Research Unit Hypertension and Cardiovascular \\ Epidemiology, KU Leuven Department of Cardiovascular Sciences, University of Leuven, \\ Leuven, Belgium; ${ }^{b}$ \& \& D Group VitaK, Maastricht University, Maastricht, The Netherlands
}

\section{Key Words}

Calcification · Matrix Gla protein · Microcirculation · Kidney · Vitamin K

\begin{abstract}
Vitamin-K-dependent carboxylation of matrix Gla protein (MGP) protects the macrocirculation against calcification. We recently reported in a multiethnic population study that the estimated glomerular filtration rate, a microvascular trait, decreased and the risk of chronic kidney disease increased with higher circulating levels of inactive dephospho-uncarboxylated MGP, a marker of vitamin K deficiency. These findings highlighted the possibility that vitamin $\mathrm{K}$ might have a beneficial effect on the renal microcirculation. To substantiate these epidemiological findings, we undertook a pilot study, in which we stained renal tissue samples obtained by biopsy from 2 healthy kidney donors and 4 patients with nephropathy for carboxylated and uncarboxylated MGP and calcium deposits. Three patients had renal calcifications, which were consistently associated with carboxylated and uncarboxylated MGP. Normal renal tissue was devoid of microcalcifications and staining for carboxylated and uncarboxylated MGP. Pending confirmation in a larger study covering a wider range of renal pathologies, these histopathological findings suggest that MGP might inhibit calcification not only in large arteries, as was known before, but in renal tissue as well, thereby highlighting potentially new avenues for promoting renal health, for instance by vitamin $\mathrm{K}$ supplementation.
\end{abstract}


Wei et al.: Vitamin-K-Dependent Protection of the Renal Microvasculature:

Histopathological Studies in Normal and Diseased Kidneys

\section{Introduction}

Vascular smooth muscle cells synthesize matrix Gla protein (MGP), a small secretory protein $(11 \mathrm{kDa})$, which contains $5 \gamma$-carboxyglutamate (Gla) amino acid residues [1]. Activation of MGP requires two posttranslational modifications: the vitamin-K-dependent $\gamma$-glutamate carboxylation and serine phosphorylation [2]. Higher circulating levels of dephospho-uncarboxylated MGP (dp-ucMGP) indicate vitamin K deficiency [3], whereas total uncarboxylated MGP (t-ucMGP) decreases with prevalent vascular calcification [4, 5].

Carboxylated MGP is a potent inhibitor of arterial calcification [2]. In patients with diabetes [6], renal dysfunction [7], or macrovascular disease [8], inactive dp-ucMGP behaves as a circulating biomarker associated with cardiovascular risk [6], more severe illness [7], or higher mortality [8]. In the Flemish Study on Environment, Genes and Health Outcomes (FLEMENGHO), circulating dp-ucMGP predicted total and cardiovascular mortality [9]. More recently, we assessed the association of renal microvascular function as exemplified by the estimated glomerular filtration rate (eGFR) with both circulating dp-ucMGP and t-ucMGP in a multiethnic population study [10]. In the Flemish population, with adjustments applied for covariables, eGFR measured on a continuous scale was inversely associated with dp-ucMGP and positively with t-ucMGP. Furthermore, in categorical analyses, the risk of moving up one stage in the classification of chronic kidney disease increased with higher dp-ucMGP [10]. We confirmed the inverse association of eGFR with dp-ucMGP in black South Africans [10]. Based on these epidemiological findings [10] and the incidental observation that in skin biopsies microvascular calcifications co-localized with MGP (fig. 1), we undertook a pilot study, in which we stained renal biopsies collected from 4 patients with renal dysfunction and 2 healthy kidney donors for carboxylated MGP (cMGP) and uncarboxylated MGP (ucMGP) and calcium deposits.

\section{Methods}

Collection of Tissue Samples

The study complied with the Helsinki declaration for research in human subjects [11] and received approval from the local ethics committee. Kidney biopsies of 4 patients with chronic kidney disease and 2 healthy kidney donors were selected from the biobank at the University Hospital Gasthuisberg, Leuven, Belgium.

\section{Histopathological Studies}

Renal tissue samples were fixed in $4 \%$ formaldehyde/phosphate-buffered saline, embedded into paraffin and cut into 4- $\mu$ m-thick sections. The sections were deparaffinized, rehydrated and stained with hematoxylin and eosin (Klinipath, Duiven, The Netherlands). Slides were then mounted with Entellan (Merck, Darmstadt, Germany). Nonspecific background immunostaining was blocked with $10 \%$ peroxidase and rabbit serum (10\% TBST; Tris-buffered saline; 0.1\% Tween-20; 1\% bovine serum albumin).

The slides were also immunohistochemically stained for MGP using conformation-specific monoclonal mouse antibodies directed against cMGP ( $4 \mathrm{mg} / \mathrm{l}$ ) and ucMGP (4 mg/l). All antibodies were diluted in blocking reagent (Tris-buffered saline; $0.1 \%$ Tween-20; $1 \%$ bovine serum albumin). Horse radish peroxidase-conjugated rabbit anti-mouse IgG (1:100; Dako, Glostrup, Denmark) was applied as the secondary antibody. Antibodies were visualized with a peroxidase substrate kit (Vector SK-4805, Vector Laboratories, Burlingame, Calif., USA). Sections were counterstained with hematoxylin and mounted with coverslips. Calcium deposits were visualized by von Kossa staining after the tissue sections had been deparaffinized, rehydrated and incubated with $1 \%$ silver nitrate for $5 \mathrm{~min}$. After washing, calcium was developed with sodium formaldehyde for $1 \mathrm{~min}$ and excess silver nitrate was removed by sodium thiosulfate. Sections were counterstained with nuclear fast red and mounted with coverslips. 


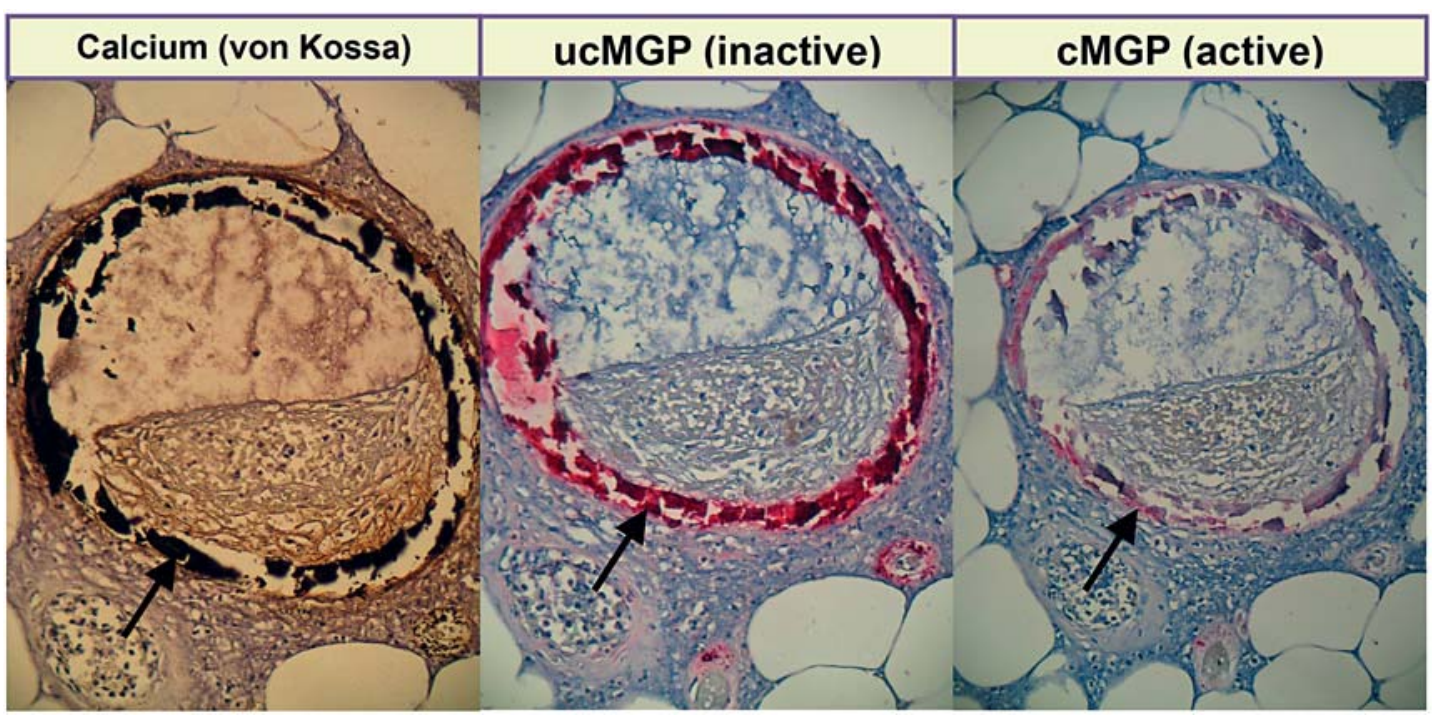

Fig. 1. Staining of skin microvessels. Arrows refer to the positive staining. Microvascular calcifications (black: von Kossa staining) co-localize with MGP; ucMGP and cMGP indicate uncarboxylated (inactive: dark red) and carboxylated (active: pink) MGP.

\section{Results}

\section{Characteristics of Participants}

All patients ( 3 female) and donors ( 1 female) were white. The patients' age averaged (SD) 68.4 (0.4) years (range, 67.9-68.7). Mean serum creatinine concentration was $231.8 \mu \mathrm{mol} / \mathrm{l}$ (range, 104.3-542.8). The underlying cause of renal failure was primary amyloidosis in 1 patient, interstitial nephritis secondary to phenacetin abuse in 2 , and renal insufficiency attributable to radiocontrast-induced nephropathy in 1 . Age and serum creatinine in the 2 healthy kidney donors were 66 and 71 years, and 50.4 and $85.7 \mu \mathrm{mol} / \mathrm{l}$, respectively.

\section{Histopathological Studies}

None of the tissue samples of healthy donors showed calcifications or immunohistochemical staining for ucMGP or cMGP. Among patients with renal dysfunction, 3 had renal calcifications in the cortical interstitium and in the deeper layers of the vascular walls with co-localization of ucMGP or cMGP. The von Kossa staining in the 4th patient was negative, with sparse weak staining for ucMGP and at the same loci even fainter staining for cMGP. Figure 2 illustrates the exemplary histopathological findings in 1 normal donor and 1 patient with interstitial nephritis due to phenacetin abuse.

\section{Discussion}

To our knowledge, our study is the first to assess the presence of both inactive ucMGP and activated cMGP in association with microcalcifications in samples of renal tissue obtained by biopsy from patients with chronic kidney disease and from healthy kidney donors. In line with the underlying hypothesis, our pilot study demonstrated that irrespective of the underlying disease process ucMGP and cMGP consistently colocalized with microcalcifications in diseased kidneys. In contrast, normal renal tissue was devoid of microcalcifications and hence 
Fig. 2. Histopathological findings in a healthy donor and a patient with interstitial nephritis. Tissue samples (magnification $\times 100$ ) were stained with hematoxylin and eosin (HE), conformationspecific antibodies directed against cMGP and ucMGP matrix Gla protein and for calcium deposits (von Kossa staining). Black arrows point to glomeruli and blue arrows to a microvessel. Healthy donor tissue was negative for calcification, cMGP and ucMGP. Tissue from the patient with interstitial nephritis revealed calcium deposits (black: von Kossa staining), which co-localized with cMGP and ucMGP.

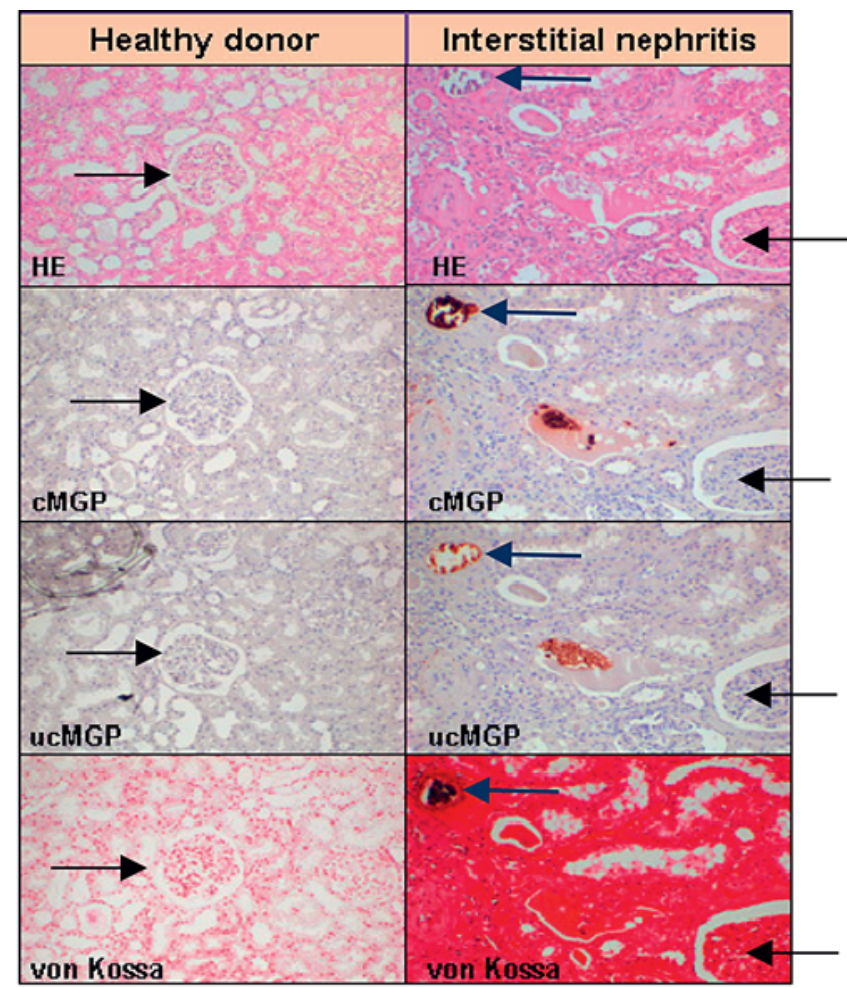

staining for both MGP moieties. These observations in the renal microcirculation are consistent with findings in mice and human endothelial cells [12] and the human macrocirculation [5].

Mice lacking the MGP gene die within 2 months because of widespread arterial calcification that leads to disintegration and rupture of the arterial wall and massive bleeds $[13,14]$. Selectively reintroducing MGP expression in the liver of the MGP-deficient mice results in circulating MGP levels 6- to 10-fold higher than in wild-type animals [15]. The MGP originating from the transgene conserved its biological activity in vitro, but did not inhibit arterial calcification [15]. Thus, MGP is locally synthesized and activated in the arterial wall and prevents arterial calcification through local effects in the arterial wall.

High levels of plasma dp-ucMGP indicate poor vitamin K status [3]. For instance, among 60 middle-aged healthy volunteers randomized in a placebo-controlled double-blind trial, circulating dp-ucMGP dropped dose-dependently by 31 and $46 \%$ in response to supplementation with 180 and $360 \mu \mathrm{g}$ of menaquinone-7 (vitamin $\mathrm{K}_{2}$ ) daily for 12 weeks, whereas no changes occurred in t-ucMGP ( $\mathrm{p}=0.23$ ) [3]. Along similar lines, among 174 patients with chronic kidney disease, stages 3-5, the criteria for vitamin K deficiency were met in 6, 60 and $97 \%$ based on plasma levels of phylloquinone, percent of uncarboxylated osteocalcin, or proteins induced by vitamin Kabsence (des- $\gamma$-carboxy-prothrombin), respectively [16]. Poor vitamin $\mathrm{K}$ status in patients with chronic kidney disease or on hemodialysis not only results from higher needs of activation of MGP in the presence of the high risk of arterial calcification, but also from lower dietary intake compared with healthy controls [17]. By far, the most extensive vascular calcifications occur in patients with chronic kidney disease $[18,19]$. In keeping with our current findings, impaired inhibition of calcification might be a major player underlying the high risk of arterial calcification, macrovascular complications, and ultimately death in patients with chronic kidney disease [19]. Our current findings extend these previous findings to the renal microcirculation. 
t-ucMGP, in the literature often referred to as ucMGP, predominantly consists of phosphorylated MGP and at variance with dp-ucMGP is not a biomarker of vitamin K status, but lower levels reflect more extensive cardiovascular calcifications [4, 5]. t-ucMGP accumulates at arterial calcification sites, possibly by binding through its negatively charged phosphoserine residues $[4,5]$. Upregulation of MGP transcription in response to vascular stress influences circulating t-ucMGP levels [4]. In 40 patients on hemodialysis, the mean t-ucMGP level was significantly lower than in healthy age-matched controls. Additionally, higher coronary calcification scores determined by multislice computed tomography were associated with lower t-ucMGP levels $(r=-0.41 ; p=0.009)$. This correlation persisted after adjustment for age, dialysis vintage, and high-sensitivity C-reactive protein as a marker of inflammation [4]. In a cross-sectional study, Parker et al. [20] investigated the association of kidney function with t-ucMGP among 842 outpatients with stable cardiovascular disease. The eGFR correlated significantly and positively with t-ucMGP in unadjusted or multivariable adjusted analyses [20].

Our current findings must be interpreted within the context of its limitations. As a first approach, we deliberately chose to undertake our histopathological studies in patients with renal pathologies of different etiology. Moreover, the sample size was small only including 4 patients and 2 controls. Future studies should aim at consolidating our current findings in a larger number of tissue samples taken from patients with diverse renal diseases diagnosed according to the state of the art, if possible with a longitudinal component to establish the timeline between progression of renal dysfunction and the increment in renal microvascular calcification. Second, we collected the renal tissue samples from the biobank available at the Leuven University Hospital. Due to this retrospective design we did not have information on the circulating levels of dp-ucMGP and t-ucMGP prior to or concurrent with the biopsies.

If confirmed, our findings have clinical implications. High levels of plasma dp-ucMGP are a proxy for vitamin $\mathrm{K}$ deficiency $[3,21]$. Levels ranging from $\sim 1.4$ to $\sim 4.6 \mu \mathrm{g} / \mathrm{l}$ are probably optimal in terms of the risk of mortality and macrovascular cardiovascular complications [9]. In white Flemish people, a $4.6 \mu \mathrm{g} / \mathrm{l}$ threshold corresponded with the 65 th percentile of the dp-ucMGP distribution, indicating that $35 \%$ of the general population in Belgium might be vitamin $\mathrm{K}$ deficient. The recommended dietary allowance for vitamin $\mathrm{K}$ is $1 \mu \mathrm{g} / \mathrm{kg}$ of body weight per day, which is sufficient to ensure normal hemostasis [22]. However, in apparently healthy subjects, a substantial fraction of MGP remains in the uncarboxylated forms [5]. This raises the question as to whether the present recommended dietary allowance for vitamin $\mathrm{K}$ intake is sufficient to prevent vascular disease. Vitamin K supplementation reduces aortic pulse wave velocity in healthy postmenopausal women [23]. Assuming reversibility, our current findings extend the protective role of vitamin $\mathrm{K}$ from the macrocirculation to the microcirculation and renal function, revealing an untapped potential for prevention by supplementation, for instance by biologically enriched fermented vegetable or dairy products. Moreover, vitamin K has a very wide safety range, irrespective of whether the source are leafy vegetables (phylloquinone; vitamin $\mathrm{K}_{1}$ ) [22] or fermented foods (menaquinones; vitamin $\mathrm{K}_{2}$ ) [22].

In conclusion, the histopathological findings in our current pilot study suggest that active MGP might inhibit calcification not only in large arteries, as was well known before [23], but also in renal tissue. These findings highlight potentially new avenues for promoting renal health, for instance by increasing the dietary intake of vitamin K either by supplementation or by increasing the intake of nutrients rich in vitamin $\mathrm{K}$. 
Wei et al.: Vitamin-K-Dependent Protection of the Renal Microvasculature:

Histopathological Studies in Normal and Diseased Kidneys

\section{Acknowledgements}

The European Union (HEALTH-2011.2.4.2-2-EU-MASCARA, HEALTH-F7-305507 HOMAGE and the European Research Council Advanced Researcher Grant-2011-294713-EPLORE) and the Fonds voor Wetenschappelijk Onderzoek Vlaanderen, Ministry of the Flemish Community, Brussels, Belgium (G.0881.13 and G.088013) currently support the Studies Coordinating Centre in Leuven. The authors gratefully acknowledge the contribution of the nurses working at the examination center (Linda Custers, Marie-Jeanne Jehoul, Daisy Thijs, and Hanne Truyens) and the clerical staff at the Studies Coordinating Centre (Vera De Leebeeck and Renilde Wolfs).

\section{Disclosure Statement}

N.A.E. Drummen, M. Herfs, C. van't Hoofd and C. Vermeer are employees of R\&D Group VitaK. The other authors declare no conflict of interest.

\section{References}

1 Hackeng TM, Rosing J, Spronk HM, Vermeer C: Total chemical synthesis of human matrix Gla protein. Protein Sci 2001;10:864-870.

-2 Schurgers LJ, Cranenburg ECM, Vermeer C: Matrix Gla-protein: the calcification inhibitor in need of vitamin K. Thromb Haemost 2008;100:593-603.

-3 Dalmeijer GW, van der Schouw YT, Magdeleyns E, Ahmed N, Vermeer C, Beulens JW: The effect of menaquinone-7 supplementation on circulating species of matrix Gla protein. Atherosclerosis 2012;225:397-402.

-4 Cranenburg EC, Brandenburg VM, Vermeer C, Stenger M, Muhlenbruch G, Mahnken AH, Gladziwa U, Ketteler M, Schurgers LJ: Uncarboxylated matrix Gla protein (ucMGP) is associated with coronary artery calcification in haemodialysis patients. Thromb Haemost 2009;10:359-366.

-5 Schurgers LJ, Teunissen KJ, Knapen MHJ, Kwaijtaal M, van Diest R, Appels A, Reutelingsperger CP, Cleutjens JP, Vermeer C: Novel conformation-specific antibodies against matrix gamma-carboxyglutamic acid (Gla) protein: undercarboxylated matrix Gla protein as marker for vascular calcification. Arterioscler Thromb Vasc Biol 2005;25:1629-1633.

-6 Dalmeijer GW, van der Schouw YT, Magdeleyns EJ, Vermeer C, Verschuren WM, Boer JM, Beulens JW: Matrix Gla protein species and risk of cardiovascular events in type 2 diabetic patients. Diabet Care 2013;36:37663771.

-7 Schurgers LJ, Barreto DV, Barreto FC, Liabeuf S, Renard C, Magdeleyns EJ, Vermeer C, Choukroun G, Massy ZA: The circulating inactive form of matrix gla protein is a surrogate marker for vascular calcification in chronic kidney disease: a preliminary report. Clin J Am Soc Nephrol 2010;5:568-575.

-8 Mayer O Jr, Seidlerová J, Bruthans J, Filipovský J, Timoracká K, Vanek J, Cerná L, Wohlfart P, Cífková R, Theuwissen E, Vermeer C: Desphospho-uncarboxylated matrix Gla-protein is associated with mortality risk in patients with chronic stable vascular disease. Atherosclerosis 2014;235:162-168.

-9 Liu YP, Gu YM, Thijs L, Knapen MHJ, Salvi E, Citterio L, Petit T, Delli Carpini S, Zhang Z, Jacobs L, Jin Y, Barlassina C, Manunta P, Kuznetsova T, Verhamme P, Struijker-Boudier HA, Cusi D, Vermeer C, Staessen JA: Inactive matrix Gla protein is causally related to adverse health outcomes: a Mendelian randomization study in a Flemish population. Hypertension 2015;65:463-470.

$\checkmark 10$ Wei FF, Drummen NEA, Schutte AE, Thijs L, Jacobs L, Petit T, Yang WY, Smits W, Zhang ZY, Gu YM, Kuznetsova T, Verhamme P, Allegaert K, Schutte R, Lerut E, Evenpoel P, Vermeer C, Staessen JA: Vitamin K dependent protection of renal function in multi-ethnic population studies. EBioMedicine 2016;4:162-169.

-11 World Medical Association: World Medical Association Declaration of Helsinki. Ethical principles for medical research involving human subjects. JAMA 2013;310:2191-2194.

12 Yao Y, Jumabay M, Ly A, Radpavar M, Cubberly MR, Boström KI: A role of the endothelium in vascular calcification. Circ Res 2013;113:495-504.

13 Schafer C, Heiss A, Schwarz A, Westenfeld R, Ketteler M, Floege J, Muller-Esterl W, Schinke T, Jahnen-Dechent W: The serum protein alpha 2-Heremans-Schmid glycoprotein/fetuin-A is a systematically acting inhibitor of ectopic calcification. J Clin Invest 2003;112:357-366.

14 Luo G, Ducy P, McKee MD, Pinero GJ, Loyer E, Behringer RR, Karsenty G: Spontaneous calcification of arteries and cartilage in mice lacking matrix GLA protein. Nature 1997;386:78-81.

15 Murshed M, Schinke T, McKee MD, Karsenty G: Extracellular matrix mineralization is regulated locally; different roles of two gla-containing proteins. J Cell Biol 2004;165:625-630.

16 Holden RM, Morton AR, Garland JS, Pavlov A, Day AG, Booth SL: Vitamins K and D status in stages 3-5 chronic kidney disease. Clin J Am Soc Nephrol 2010;5:590-597. 
17 Cranenburg ECM, Schurgers LJ, Uiterwijk HH, Beulens JW, Dalmeijer GW, Westerhuis R, Magdeleyns J, Herfs M, Vermeer C, Laverman GD: Vitamin K intake and status are low in hemodialysis patients. Kidney Intern 2012;82:605-610.

18 Lanzer P, Boehm M, Sorribas V, Thiriet M, Janzen J, Zeller T, St Hilaire C, Shanahan C: Medial vascular calcification revisited: review and perspectives. Eur Heart J 2014;35:1515-1525.

19 Moe SM, Chen NX: Pathophysiology of vascular calcification in chronic kidney disease. Circ Res 2004;95:560567.

20 Parker BD, Ix JH, Cranenburg ECM, Vermeer C, Whooley MA, Schurgers LJ: Association of kidney function and uncarboxylated matrix Gla protein: data from the Heart and Soul Study. Nephrol Dial Transplant 2009;24: 2095-2101.

-21 Westenfeld R, Krueger T, Schlieper G, Cranenburg EC, Magdeleyns EJ, Heidenreich S, Holzmann S, Vermeer C, Jahnen-Dechent W, Ketteler M, Floege J, Schurgers LJ: Effect of vitamin $\mathrm{K}_{2}$ supplementation on functional vitamin K deficiency in hemodialysis patients: a randomized trial. Am J Kidney Dis 2012;59:186-195.

22 Weber P: Vitamin K and bone health. Nutrition 2001;17:880-887.

-23 Knapen MHJ, Braam LAJLM, Drummen NE, Bekers 0, Hoeks APG, Vermeer C: Menaquinone-7 supplementation improves arterial stiffness in healthy postmenopausal women. Thromb Haemost 2015;113:1135-1144. 